\title{
Antithrombotic Strategies in Perioperative Period
}

\author{
MD NAZMUL HASAN, MD ABU SIDDIQUE, SAJAL KRISHNA BANERJEE, SYED ALI AHSAN, \\ A KM FAZLUR RAHMAN, CHOUDHURY MESHKAT AHMED, MOHAMMAD FAISAL IBN KABIR, \\ MD HARISUL HOQUE \\ Department of Cardiology, Bangabandhu Sheikh Mujib Medical University (BSMMU), Dhaka. \\ Address for Correspondence: Dr Md Nazmul Hasan, Resident, Department of Cardiology, BSMMU \\ Email: drnhn45cmc@yahoo.com
}

\begin{abstract}
:
Antithrombotic therapy (Warfarin, Aspirin,Clopidogrel etc.) are routinely used in prevention and treatment of various cardiovascular diseases. In patients who are having a surgical or other invasive procedure, interruption of antithrombotic therapy is typically required to minimize the risk for perioperative bleeding. It involves balancing the risk of periprocedural bleeding with continued treatment against the thrombotic risk with suspension of treatment and use of bridging anticoagulation therapy. The need for bridging is driven by patients' estimated risk for thromboembolism and the bleeding risk of invasive procedure. Treatment with subcutaneous low-molecular-weight heparin or intravenous unfractionated heparin at a therapeutic dose before and after the procedure recommended as bridging anticoagulation therapy.
\end{abstract}

Key Wards : Antithrombotic drugs, Perioperative period, Strategy

\section{Introduction:}

Antithrombotic therapy should be suspended in a patient who will have an an invasive procedure requires $q$ balance between the risk of periprocedural bleeding with continued treatment against the thrombotic risks with suspension of treatment and use of bridging anticoagulation therapy.In general, a patient undergoing a procedure that is associated with a low risk of bleeding (low-risk procedure) can safely continue antithrombotic therapy. Conversely, a patient undergoing a high-risk procedure should temporarily discontinue antithrombotic agents safely if the patient is at low risk for a thromboembolic event (low-risk patient). The decision-making is challenging when patients at moderate-to-high risk for thromboembolic events undergo high-risk procedures. Management also differs between elective and emergency procedures. Appropriate decision making requires knowledge of thrombotic risk ,procedurerelated bleeding risk, concepts of bridging anticoagulation therapy,timing of cessation and initiation of antithrombotic therapy again ${ }^{1}$.

Suggested Risk Stratification: Venous Thromboembolism²

High Risk ${ }^{3}$

-Recent VTE ( $<3$ months ago)

-Severe thrombophilia (eg, antiphospholipid antibodies)

Moderate Risk

-VTE within the past 3-12 months

-Non severe thrombophilia (eg, heterozygous factor V mutation)

-Recurrent VTE

-Active cancer (treated within 6 months or palliative)
Low Risk

Prior VTE $>12$ months ago and no other risk factors

Suggested Risk Stratification: Mechanical Heart Valvest

High Risk

-Any mitral valve prosthesis

-Older (caged-ball or tilting disc) aortic valve prosthesis

-Recent (within 6 months) stroke or TIA

Moderate Risk

Bileaflet aortic valve and at least one of the followings:

Atrial fibrillation, prior stroke or transient ischemic attack, hypertension, diabetes, congestive heart failure, age $>75$ years

Low Risk

Bileaflet aortic valve without atrial fibrillation and no other risk factors for stroke

Suggested Risk Stratification :Atrial Fibrillationv

High Riskw

CHADS $_{2}$ score $=5-6$

Recent (within 3 months) stroke or TIA

Rheumatic valvular heart disease

Moderate Risk

CHADS $_{2}$ score $=3-4$

Low Risk

$\mathrm{CHADS}_{2}$ score $=0$-2 and no prior stroke or TIA

Suggested Risk Stratification: Coronary Stents

-Premature discontinuation of antiplatelet therapy in anticipation of a surgical or other invasive procedure may lead to Stent thrombosis, Precipitation of myocardial 
infarction, Mortality rate of $50 \%$ or higher ,the risk of stent thrombosis differs between bare-metal stents and drug-eluting stents.

-The risk of thrombosis is highest within 6 weeks after the placement of a bare-metal stent and within 3 to 6 months after the placement of a drug-eluting stent.Antiplatelet therapy is required for at least 1 month after placement of a bare-metal stent and for 1 year after placement of a drugeluting stent ${ }^{1} \mathrm{p}$.

-The optimal duration of dual antiplatelet therapy for patients with coronary stents remains unknown. However, recent studies suggest that 6 to 12 months may be appropriate, with little to gain from a longer duration.

Risk Stratification for Bleeding

High bleeding-risk surgeries/procedures include:

- Urologic surgery/procedures: TURP, bladder resection or tumor ablation, nephrectomy or kidney biopsy (untreated tissue damage after TURP and endogenous urokinase release)

- Pacemaker or ICD implantation (separation of infraclavicular fascia and no suturing of unopposed tissues may lead to hematoma)

- Colonic polyp resection, especially $>1-2 \mathrm{~cm}$ sessile polyps (bleeding occurs at transected stalk after hemostatic plug release)

- Vascular organ surgery: thyroid, liver, spleen

- Bowel resection (bleeding may occur at anastomosis site)

- Major surgery involving considerable tissue injury: cancer surgery, joint arthroplasty, reconstructive plastic surgery

- Cardiac, intracranial or spinal surgery (small bleeds can have serious clinical consequences)

Is interruption of antithrombotic therapy in the perioperative period needed?

-In patients who are having a major surgical or other major invasive procedure, interruption of antithrombotic therapy is typically required to minimize the risk for perioperative bleeding. Continuation of vitamin K antagonist (VKA) therapy or aspirin in the perioperative period confers an increased risk for bleeding.

-In patients who are undergoing minor surgical or invasive procedure (eg, dental, skin, or cataract), interruption of antithrombotic therapy may not be required.
If antithrombotic therapy is interrupted before surgery, is "bridging Anticoagulation" needed?

The need for bridging is driven by patients' estimated risk for thromboembolism (TE):

-In high-risk patients, the need to prevent TE will dominate management irrespective of bleeding risk; the potential consequences of TE may justify bridging $x$.

-In moderate-risk patients, a single perioperative strategy is not dominant and management will depend on individual patient risk assessment

In low-risk patients, the need to prevent TE will be less dominant and bridging may be avoided

-In all patients, judicious use of postoperative bridging is needed to minimizing bleeding that would have the undesired effect of delaying resumption of antithrombotic therapy after surgery.

\section{What are bridging anticoagulation regimens?}

-A "high-dose” (therapeutic-dose) regimen involves giving a dose similar to that used to treat acute VTE or ACS (eg, enoxaparin, $1 \mathrm{mg} / \mathrm{kg}$ BID or $1.5 \mathrm{mg} / \mathrm{kg}$ QD, dalteparin $100 \mathrm{IU} / \mathrm{kg}$ BID or $200 \mathrm{IU} / \mathrm{kg}$ QD, tinzaparin $175 \mathrm{IU} / \mathrm{kg}$ $\mathrm{QD}, \mathrm{IV}$ UFH to attain aPTT 1.5- to 2-times the control aPTT).

-A “low-dose” (prophylactic-dose) regimen involves giving a dose used, typically, to prevent postoperative VTE (eg, enoxaparin 30 mg BID or 40 mg QD, dalteparin 5000 IU QD, UFH 5000-7500 IU BID)

-An “intermediate-dose” regimen has been recently studied for bridging and is intermediate in anticoagulant intensity between a high-dose and low-dose regimen (eg, enoxaparin $40 \mathrm{mg}$ BID).

\section{Bridging anticoagulation therapy}

-In most cases, bridging anticoagulation therapy is used in patients receiving warfarin.Once warfarin has been discontinued and the international normalized ratio (INR) falls below the therapeutic range,intravenous unfractionated heparin or subcutaneous low-molecular weight heparin is administered for 3 to 5 days

-The heparin agent is withdrawn before the procedure, with the timing based on whether unfractionated heparin or lowmolecular-weight heparin is usedis usually readministered 48 hours after the procedure, if hemostasis is secured

-Stop warfarin 5 days before a high-risk procedurewhen the INR falls below the therapeutic range, begin low molecular- weight heparin at a therapeutic dosey . 
-For patients with a mechanical heart valve or atrial fibrillation, use enoxaparin at a dose of $1 \mathrm{mg}$ per kilogram of body weight, administered every 12 hours

-The final dose (either enoxaparin at a dose of $1 \mathrm{mg}$ per kilogram) should be administered 24 hours before the procedure

-Check the INR on the morning of the procedure.

-Restart warfarin therapy immediately after the procedure if hemostasis is secured

-Reinstitute treatment with subcutaneous low-molecularweight heparin or intravenous unfractionated heparin at a therapeutic dose (without bolus) 48 hours after the procedure if no bleeding has occurredwith the exception that for patients undergoing endoscopic sphincterotomy , heparin therapy should be initiated after 72 hours

-Discontinue heparin therapy when the INR is in the therapeutic range (approximately 5 days later).

Timing of Cessation of Antithrombotic Therapy

Warfarin

-Bleeding risk not to be elevated when the INR is 1.5 or less assumed to be elevated when the INR is more than 2.0.Thus, an INR of 1.5 or less is considered safe for highrisk procedures

-An INR of 2.0 to 3.5 corresponds to therapeutic anticoagulation, and $93 \%$ of patients with an INR within this range have an INR of less than 1.5 approximately 5 days after warfarin therapy has been discontinued.

-The INR should be obtained within 24 hours before the procedure and corrected with vitamin $\mathrm{K}$, if needed, except in the presence of mechanical heart valves

-When warfarin therapy is continued through the procedure, it is important that the INR before the procedure not be supratherapeutic

-Adjust warfarin doses over a period of 5 days to aim for an INR of approximately 2.5 by the time of the procedure.

Heparin

-Unfractionated heparin administered intravenously has a half-life of 60 to 90 minutes, and Anticoagulant effects dissipate 3 to 4 hours after discontinuation thus, the infusion is stopped 4 to 6 hours before high-risk proceduresy .

-Low molecular- weight heparin is administered subcutaneously at therapeutic doses for bridging and for the treatment of venous thromboembolism, with reduced doses for the prevention of venous thromboembolism in low-risk patients.

-The half-life of these agents is approximately 4 hours, and the last dose should be given 24 hours before the anticipated procedure at $50 \%$ of the total daily dose (i.e., enoxaparin at a dose of $1 \mathrm{mg}$ per kilogram or dalteparin at a dose of 100 IU per kilogram).

Resumption of Antithrombotic Therapy

-In patients receiving bridging therapy, heparin at a therapeutic dose should be withheld for 48 hours after the procedure.

-warfarin therapy can be reinstituted the evening of the day on which the procedure is performed

-Clopidogrel administered at maintenance doses has a delayed onset of action, and treatment can therefore be reinitiated within 24 hours after the procedure

-Treatment with other antiplatelet agents, includingaspirin, can be reinitiated within 24 hours.

Case Vignette No. 1

A 68-year-old woman receiving chronic warfarin for recurrent DVT (most recent was 1 year ago) will undergo two dental extractions that will include local anesthetic injections... Patient has concerns about dental bleeding...

Recommendation:In patients who require minor dental surgery and are receiving VKA therapy, suggesting either continuing VKA with co-administration of an oral prohemostatic agent or stopping VKAs 2-3 days before the procedure instead of alternative strategies (Grade 2C) ${ }^{1}$.

Recommendation:In patients who require minor skin procedures and are receiving VKA therapy, suggests continuing VKAs around the time of the procedure and optimizing local hemostasis instead of other strategies (Grade 2C).

Recommendation:In patients who require cataract surgery and are receiving VKA therapy, suggests continuing VKAs around the time of the surgery instead of other strategies (Grade 2C).

Case Vignette No. 2

A 54-year-old man with a mechanical mitral valve replacement on long-term warfarin therapy is scheduled for total hip replacement.Concerns About Post-Op Bleeding with Bridging...

Recommendation: In patients who require temporary interruption of a VKA before surgery, recommendation is stopping VKAs approximately 5 days before surgery 
instead ofstopping VKAs a shorter time before surgery (Grade 1C).

Recommendation: In patients who require temporary interruption of a VKA before surgery, recommendation is resuming VKAs approximately 12-24 hours after surgery (evening of or next morning) and when there is adequate hemostasis instead of later resumption of VKAs (Grade 2C).

Recommendation: In patients with a mechanical heart valve, atrial fibrillation or VTE at high risk for TE,suggests bridging anticoagulation instead of no bridging during interruption of VKA therapy (Grade 2C).

Recommendation: In patients who are receiving bridging anticoagulation with therapeutic-dose SC LMWH, suggests administering the last preoperative dose of LMWH approximately $24 \mathrm{~h}$ before surgery instead of 12 h before surgery (Grade 2C).

Recommendation: In patients who are receiving bridging anticoagulation with therapeutic-dose SC LMWH and are undergoing high bleeding-risk surgery, suggests resuming therapeutic-dose LMWH 48-72 h after surgery instead of resuming LMWH within $24 \mathrm{~h}$ after surgery (Grade 2C).

\section{Case Vignette No. 3}

A 69-year-old man with chronic atrial fibrillation and hypertension $\left(\mathrm{CHADS}_{2}\right.$ score $=1$ ) is undergoing an abdominal surgery for cancer.

Recommendation: In patients with a mechanical heart valve, atrial fibrillation or VTE at low-risk for TE, suggests no bridging instead of bridging anticoagulation during interruption of VKA therapy (Grade 2C).

\section{Case Vignette No. 4}

66-year-old man with a drug-eluting coronary stent inserted 5 months ago following a non-ST-elevation myocardial infarction now requires surgery for removal of a parotid adenocarcinoma,He is receiving ASA $81 \mathrm{mg}$, and clopidogrel $75 \mathrm{mg}$ daily.His other cardiovascular risk factors are the following: CABG 12 years ago, hypertension, and type 2 diabetes.. Concerns about Acute Coronary Stent Thrombosis.

Recommendation: In patients with a coronary stent who are receiving dual antiplatelet therapy and require surgery, recommendation is deferring surgery for at least 6 weeks after placement of a bare-metal stent and for at least 6 months after placement of a drug-eluting stent instead of undertaking surgery within these time periods (Grade 1C).
Recommendation: In patients who require surgery within 6 weeks of placement of a bare-metal stent or within 6 months of placement of a drug-eluting stent, continuing dual antiplatelet therapy around the time of surgery instead of stopping dual antiplatelet therapy 7-10 days before surgery (Grade 2C). Aspirin therapy should never be discontinued.

Recommendation: For patients with coronary-artery stents undergoing a high-risk procedure, more than 6 weeks after the placement of a bare-metal stent or more than 6 months after the placement of a drug-eluting stent, aspirin should be continued, with thienopyridine therapy temporarily discontinued at an appropriate interval before the procedure .

Recommendation: For patients with coronary artery stents and those at high risk for cardiovascular atherosclerotic events who are undergoing a low-risk procedure full-dose antiplatelet therapy should be continued.

\section{Case Vignette No. 5}

78-year-old obese woman with a non-ST-elevation myocardial infarction 2 years ago is seen preoperatively prior to inguinal hernia repair,CAD treated medically, no angiography,receiving ASA 81 mg,other cardiovascular risk factors are the following: hypertension, dyslipidemia, glucose intolerance

Recommendation:In patients at moderate-to-high riskforcardiovascular events who are receiving ASA therapy and require non-cardiac surgery, we suggest continuing ASA around the time of surgery instead of stopping ASA 7-10 days before surgery (Grade 2C). Recommendation: In patients at low risk for cardiovascular events who are receiving ASA therapy, we suggest stopping ASA 7-10 days before surgery instead of continuation of ASA (Grade 2C).

\section{Conclusion:}

To eliminate effect of antithrombotic therapy before surgery, treatment should be stopped before surgery ( 5 days for warfarin, 7-10 days for antiplatelet drug) to minimize bleeding risk. But this bridging therapy may also become a cause of bleeding in some cases, this risk depends on anticoagulant dose (therapeutic-dose > lowdose) and proximity to surgery (higher risk if given closer to surgery). Delaying resumption of therapeutic-dose bridging (for 48-72 h after surgery), decreasing the dose or avoiding its use after surgery can mitigate the risk for bleeding. Low-dose LMWH/UFH is effective to prevent postoperative venous thromboembolism(VTE), but 
evidence is lacking that such regimens effective to prevent acute thromboembolism( ATE).

In resuming treatment after surgery, it takes 2-3 days for anticoagulant effect to begin after starting warfarin, 3-5 h for peak anticoagulant effect after starting LMWH ,3-7 days for peak inhibition of platelet aggregation after starting a maintenance dose of clopidogrel .Most surgery/ procedures done out-of-hospital and potential thromboembolic or bleeding complications occur during the initial 2 weeks after surgery while patient is at home.Close patient follow-up during early postopoperative period allows early detection and treatment of complications.

\section{Abbreviations}

aPTT activated partial thromboplastin time

ASA acetylsalicylic Acid

ATE arterial thromboembolism

CABG coronary artery bypass graft

$\mathrm{CHADS}_{2}$ congestive heart failure, hypertension, age e” 75 years, diabetes mellitus, prior stroke or transient ischemic attack

INR international normalized ratio

LMWH low-molecular-weight heparin

UFH unfractionated heparin

VKA vitamin $\mathrm{K}$ antagonist

Grade 1 Benefit >>> Risk (Procedure/ Treatment should be performed)

Grade 2 Benefit >> Risk (It is reasonable to perform Procedure/ Treatment)

Grade 1 No Benefit / Harm

Level A Multiple population studied

Level B Limited population studied

Level C Very limited population studied

\section{References:}

1. Douketis JD, Berger PB, Dunn AS, et al. ; American College of Chest Physicians The perioperative management of antithrombotic therapy: American College of Chest Physicians evidence-based clinical practice guidelines (8th edition). Chest. 2008;133(suppl 6):299S-339S.
2. Douketis JD, Foster GA, Crowther MA, et al. Clinical risk factors and timing of recurrent venous thromboembolism during the initial 3 months of anticoagulant therapy. Arch Intern Med. 2000;160(22):3431-3436.

3. Geerts WH, Pineo GF, Heit JA, et al. Prevention of venous thromboembolism: the Seventh ACCP Conference on Antithrombotic and Thrombolytic Therapy. Chest. 2004;126(suppl 3):338S-400S. 4. Lee AY, Levine MN, Baker RI, et al. ; Randomized Comparison of Low-Molecular-Weight Heparin versus Oral Anticoagulant Therapy for the Prevention of Recurrent Venous Thromboembolism in Patients with Cancer (CLOT) Investigators Low-molecular-weight heparin versus a coumarin for the prevention of recurrent venous thromboembolism in patients with cancer. N Engl J Med. 2003;349(2):146-153.

4. Cannegieter SC, Rosendaal F, Briët I. Thromboembolic and bleeding complications in patients with medical mechanical heart valve prostheses. Circulation. 1994;94(2):635-641. 6. Hering D, Piper C, Bergemann R, et al. Thromboembolic and bleeding complications following St. Jude Medical valve replacement: results of the German Experience With Low-Intensity Anticoagulation Study. Chest. 2005;127(1):53-59.

5. Tominaga R, Kurisu K, Ochiai Y, et al. A 10-year experience with the Carbomedics cardiac prosthesis. Ann Thorac Surg. 2005;79(3):784-789. 8. Gage BF, van Walraven C, Pearce L, et al. Selecting patients with atrial fibrillation for anticoagulation: stroke risk stratification in patients taking aspirin. Circulation. 2004;110(16):2287-2292.

6. Anter E, Jessup M, Callans DJ. Atrial fibrillation and heart failure: treatment considerations for a dual epidemic. Circulation. 2009;119(18):2516-2525. 10. Go AS, Hylek EM, Borowsky LH, Phillips KA, Selby JV, Singer DE. Warfarin use among ambulatory patients with nonvalvular atrial fibrillation: the anticoagulation and risk factors in atrial fibrillation (ATRIA) study. Ann Intern Med. 1999;131(12):927-934.

7. Kaatz S, Douketis JD, Zhou H, et al. Risk of stroke after surgery in patients with and without chronic atrial fibrillation.J ThrombHaemost. 2010;8(5):884-890.

8. Ansell JE. The perioperative management of warfarin therapy. Arch Intern Med. 2003;163(8):881-883. 13. Douketis JD, Bakhsh E. Perioperative management of antithrombotic therapy. Pol Arch Med Wewn. 2008;118(4):201-208. 14. Spyropoulos AC. Bridging of oral anticoagulation therapy for invasive procedures.CurrHematol Rep. 2005;4(5):405-413.

9. Dunn A. Perioperative management of oral anticoagulation: when and how to bridge. J Thromb Thrombolysis. 2006;21(1):85-89.

10. Metzler H, Kozek-Langenecker S, Huber K. Antiplatelet therapy and coronary stents in perioperative medicine: the two sides of the coin. Best Pract Res ClinAnaesthesiol 2008; 22:81-94. 